\title{
The Research on Mechanical Performance of Portal Crane Main Steel Structure Based on Sustainable Development
}

\author{
Bin Zhang ${ }^{1,}$, Guo-feng Li $^{1}$, Zheng-qiu Huang ${ }^{2}$, Yao-ting Tong ${ }^{2}$ \\ ${ }^{1}$ Shanghai Fengxian District Special Equipment Inspection Institute, 201406 Shanghai \\ ${ }^{2}$ Shanghai Institute of Special Equipment Inspection and Technical Research, 200062 Shanghai,
}

\begin{abstract}
This paper introduces an evaluation method of mechanical performance of portal crane main steel structure. The risk factors of portal crane are investigated from five aspects of strength, stiffness, corrosion, crack and local stability. Through quantification, each evaluation index is transformed into a quantitative value that is easy to identify, which directly reflects the state of the steel structure of the crane. Scientific and reasonable evaluation results are beneficial to maintenance and use of portal crane, and it can save energy and reduce emission, reduce cost and increase efficiency.
\end{abstract}

\section{Introduction}

Portal crane is developed with the development of port industry. In recent years, with the rapid development of economy, China's import and export trade continues to grow, cargo transportation is becoming more and more large, which promotes the rapid development of largescale terminals, and the accompanying gantry crane also increases. The design and manufacture of portal crane is developing towards large-scale, high-speed, automatic and intelligent road to meet the needs of the market [1].

The design life and service life of the crane is very long. In the continuous use process, the service life is increasing, and the steel structure of the portal crane is also deteriorating, which will present various defects such as fatigue cracking, corrosion, deformation and abrasion. If not found and repaired in time, various types of damage will accumulate for a long time, which will greatly reduce the residual fatigue life of the crane Life.

In order to improve the safety and reliability of crane steel structure, enterprises generally adopt the method of "regular maintenance and post maintenance". Obviously, this method is not suitable for the development of gantry crane, and also affects the working potential of the whole crane. Therefore, through the calculation or analysis of the important indexes of the portal crane steel structure, and through the dimensionless treatment, the easily recognizable evaluation value can be obtained, to Help enterprises better identify the real state of the crane. Therefore, this paper discusses the evaluation method of mechanical properties of portal crane main steel structure, which can fundamentally improve the safety of portal crane.

\section{Mechanical performance evaluation method of portal crane main steel structure}

\subsection{Seletion of evaluation index}

The portal crane will suffer from corrosion, fatigue damage or mechanical damage during its service life, which is related to the service age of the crane. Therefore, it is particularly important to detect and monitor the structural state of the crane reasonably and to ensure the safety of its structural performance with the least maintenance investment, so as to obtain the maximum economic benefits. In this paper, the strength, stiffness, corrosion, crack and local stability are selected to evaluate the metal structure of the whole machine.

\subsection{Calculation and measurement method of evalution value of each index}

\subsubsection{Strength}

Place the figure When the static stress of the structure is less than the yield limit or strength limit of the material, the static failure will not occur and the safety of the metal structure will not be affected [2]. In the strength index evaluation, the maximum static stress of the component is taken as the evaluation index, and the allowable stress of the material is used as the verification. As long as the maximum static stress is less than the allowable stress of the material, the requirements are met [3]. The formula is used for dimensionless calculation, so the evaluation value of strength index is 


$$
m_{y}=1-\frac{\sigma_{\text {measure }}}{[\sigma]}
$$

When $m_{y} \leq 0.2$, the index value is close to the allowable deflection and the stiffness is very poor;

When $0.2<m_{y} \leq 0.4$, the index value is close to the allowable deflection and the stiffness is poor;

When $0.4<m_{y} \leq 0.6$, the distance between the index value and the allowable deflection is moderate and the stiffness is general;

When $0.6<m_{y} \leq 0.8$, the index value is far away from the allowable deflection and the stiffness is strong;

When $0.8<m_{y} \leq 1.0$, the index value is far away from the allowable deflection and the stiffness is very strong.

According to the formula, the greater the ratio of the maximum static stress to the allowable stress of the material is, the weaker the strength is; otherwise, the strength is better.

Measurement method: according to the distribution diagram of stress measuring points (high stress points in the pressure area and tension area of the equipment), the inspector shall polish each point, paste the strain gauge and connect it to the strain tester, zero the crane when the boom is in the minimum range under no-load condition, lift the rated load, and record the stress value of each measuring point when the boom amplitude reaches the maximum range [4].

\subsubsection{Stiffness}

Although the stiffness of the structure cannot directly determine the bearing capacity of the structure, but too poor stiffness will affect the performance of the structure and worsen the working conditions of the structure, which will affect the bearing capacity of the structure. The state value of static stiffness index is subject to the maximum static deflection in design, and the allowable deflection is the limit value [5]. As long as the measured maximum static deflection is less than the allowable deflection, it is regarded as meeting the requirements. The allowable static deflection is selected according to the crane design code. The formula is used for dimensionless calculation, so the evaluation value of stiffness index is

$$
m_{r}=1-\frac{f_{\text {measure }}}{[f]}
$$

When $m_{r} \leq 0.2$, the index value is close to the allowable deflection and the stiffness is very poor;

When $0.2<m_{r} \leq 0.4$, the index value is close to the allowable deflection and the stiffness is poor;

When $0.4<m_{r} \leq 0.6$, the distance between the index value and the allowable deflection is moderate and the stiffness is general;
When $0.6<m_{r} \leq 0.8$, the index value is far away from the allowable deflection and the stiffness is strong;

When $0.8<m_{r} \leq 1.0$, the index value is far away from the allowable deflection and the stiffness is very strong.

According to the formula, the greater the ratio of the maximum static deflection to the allowable static deflection is, the weaker the stiffness is; otherwise, the stiffness is better.

Measurement method: mark the points where the maximum deflection may occur (boom, the hinge point of big pull rod and elephant nose bridge, bridge of elephant nose, middle of boom), measure the threedimensional coordinates of the marked points under noload and rated load by total station, and then calculate the down deflection.

\subsubsection{Corrosion}

According to the safety regulations and relevant regulations of hoisting machinery, when the section corrosion of main stressed components reaches $10 \%$ of the design thickness, if it cannot be repaired, it should be scrapped [6]. Taking the ratio of corrosion depth to raw material thickness as the evaluation value, the greater the ratio of corrosion depth to the thickness of raw material, the greater the corrosion, otherwise the less corrosion. Therefore, the evaluation value formula of corrosion index is

$$
m_{x}=1-\frac{\beta}{10 \%}
$$

When $m_{x} \leq 0.2$, corrosion is very obvious and spalling is serious;

When $0.2<m_{x} \leq 0.4$, the corrosion is obvious and there is spalling phenomenon;

When $0.4<m_{x} \leq 0.6$, the corrosion is a little obvious and the spalling is not serious;

When $0.6<m_{x} \leq 0.8$, the corrosion is not obvious and there is no peeling phenomenon;

When $0.8<m_{x} \leq 1.0$, the corrosion is slight and has little effect.

Measurement method: polish the paint at the corrosion place with polishing machine and sandpaper, and the metal luster shall be observed. Measure the thickness with a thickness gauge and record the data. Compared with the original thickness of normal parts.

\subsubsection{Crack}

Crack is the main fault of metal structure. With cracks, the metal structure will not lose its bearing capacity immediately, which is an accumulation process. So considering the actual working condition of crane, it is required to inspect the cracked parts regularly from the perspective of plant safety [7]. According to the method of calculating fatigue crack life, the residual life $t$ of 
crack is calculated. The empirical formula of inspection period is as follows

$$
T_{1}=\frac{365 T}{100}=3.65 T
$$

The crack index state value $\mathrm{T} 1$ is calculated by the formula, and the inspection period of 10 days is taken as the index limit value. The calculation formula of crack index evaluation value is [8]

$$
m_{t}=1-\frac{10}{T_{1}}
$$

When $m_{t} \leq 0.5$, the cracks are quite obvious and need to be repaired;

When $0.5<m_{t \leq 0.7}$, the cracks are obvious and need to be repaired after work;

When $0.7<m_{t \leq 0.8}$, the cracks are more obvious, so it is necessary to strengthen inspection and timely repair;

When $0.8<m_{t} \leq 0.9$, the cracks are not obvious, so it is only necessary to strengthen the monitoring and inspection;

When $0.9<m_{t \leq 1.0}$, the crack is not obvious, so the inspection should be strengthened.

\subsubsection{Local stability}

For the portal crane in use, the waviness index of main components is mainly investigated: for the compression component with local waviness, the local deflection will produce obvious stress concentration phenomenon, which makes the stress on the compression component section extremely uneven, and the maximum stress may even exceed the yield limit, so it may reduce the bearing capacity of the component. The evaluation value of main component waviness index is the ratio of limit load and nominal load. The formula is as follows:

$$
m_{b}=\frac{P_{u}}{P_{y}}
$$

When $\mathrm{MB} \leq 0.2$, the plate has a very obvious change and needs to be strengthened;

When $0.2<\mathrm{MB} \leq 0.4$, the deformation of the plate is obvious, and the bearing capacity decreases a lot;

When $0.4<\mathrm{MB} \leq 0.6$, the deformation is not obvious, and the bearing capacity decreases;

When $0.6<\mathrm{MB} \leq 0.8$, the plate has slight deformation and the bearing capacity decreases slightly;

When $0.8<\mathrm{MB} \leq 1.0$, the plate is in good condition, with very slight deformation and little influence.

Measurement method: keep a $1000 \mathrm{~mm}$ steel ruler close to the surface to be measured, then measure the maximum deformation of the part with a $300 \mathrm{~mm}$ steel ruler, measure the width of the plate, grind the plate to metallic luster, measure the thickness of the plate with a thickness gauge, and record the data.

\section{Conclusion}

In this paper, combined with the characteristics of portal crane steel structure evaluation index is easy to quantitative analysis, based on the relevant theoretical knowledge of crane metal structure safety evaluation, the method of mechanical performance evaluation of portal crane main steel structure is given.

Firstly, the risk factors of portal crane main steel structure are considered from five aspects of strength, stiffness, corrosion, crack and local stability through scientific and reasonable selection of evaluation index. Then the state value of the measurement index is measured and calculated, and then the easily identified index evaluation value is calculated.

The operation is simple, reduces the subjective human factor evaluation, and can more directly reflect the crane steel structure state, so that people have a quantitative understanding of the risk degree of the gantry crane in use. Through the introduction of this paper, it is more conducive to carry out the evaluation of the main steel structure of the portal crane, and the correct and effective evaluation can help the user to maintain the equipment reasonably, so as to achieve the effect of energy saving and emission reduction, cost reduction and efficiency increase.

\section{Acknowledgements}

I would like to express my sincere thanks to Shanghai Fengxian District Special Equipment Inspection Institute. With the help of my colleagues, I completed this paper.

Last my thanks would go to my beloved family for their loving considerations and great confidence in me all through these years. I also owe my sincere gratitude to my friends who gave me their help and time in listening to me and helping me work out my problems during the difficult course of the thesis.

\section{References}

1. Huang Hai. Research on safety evaluation method of metal structure of port crane [P]. Wuhan: Wuhan University of technology, 2008

2. Ma Rui, research on reliability of structural safety assessment of portal crane jib system [D]. Dalian Jiaotong University, 2008

3. GB / t3811.2008 code for design of cranes [S]. Beijing: China Standard Press, 2008

4. Wang Wenqin. Safety evaluation based on metal structure of shipyard portal crane $[\mathrm{J}]$. Science and technology wind, 2012 (22): 30.30 
5. He Yali. Research on fatigue life analysis and safety evaluation method of overhead traveling crane [D]. Beijing University of chemical technology, 2014

6. Research and application of safety evaluation method for portal crane [D]. Zhejiang University of technology, 2014

7. Dong Liangcai, MI Weijian. Fatigue crack growth control of container crane based on reliability [J]. Acta Sinica, 2007, 5 (4): 453-460

8. Ding Jun, Huang Hai, Yuan Dongsheng, Zhang weipeng. Safety evaluation of metal structure of port crane [J]. Port handling, 2011 (5): 7-11 